\title{
Impact of the solvent capacity constraint on $E$. coli metabolism
} Alexei Vazquez*†1, Qasim K Beg ${ }^{\dagger 2,7}$, Marcio A deMenezes ${ }^{3}$, Jason Ernst ${ }^{4}$, Ziv Bar-Joseph ${ }^{4}$, Albert-László Barabási ${ }^{5}$, László G Boros ${ }^{6}$ and Zoltán N Oltvai*2

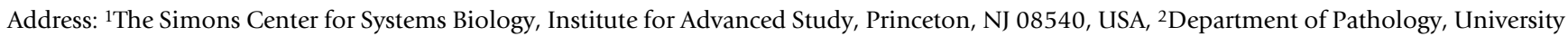
of Pittsburgh, Pittsburgh, PA, 15261, USA, 3Instituto de Física, Universidade Federal Fluminense, Rio de Janeiro, 24210, Brazil, ${ }^{4}$ Machine Learning Department, Carnegie-Mellon University, Pittsburgh, PA, 15217, USA, ${ }^{5}$ Department of Physics and Center for Complex Networks Research, University of Notre Dame, South Bend, IN 46556, USA, ${ }^{6}$ SiDMAP, LLC and the UCLA School of Medicine, Los Angeles, CA 90064, USA and ${ }^{7}$ Department of Biomedical Engineering, Boston University, Boston, MA 02215, USA

Email: Alexei Vazquez* - vazquez@ias.edu; Qasim K Beg - qasimbeg@bu.edu; Marcio A deMenezes - marcio@if.uff.br; Jason Ernst - jernst@cs.cmu.edu; Ziv Bar-Joseph - zivbj@cs.cmu.edu; Albert-László Barabási - alb@nd.edu;

László G Boros - lboros@sidmap.com; Zoltán N Oltvai* - oltvai@pitt.edu

* Corresponding authors †Equal contributors
\end{abstract}

Published: 23 january 2008

BMC Systems Biology 2008, 2:7 doi:10.1 186/1752-0509-2-7
Received: 29 August 2007

Accepted: 23 January 2008

This article is available from: http://www.biomedcentral.com/l752-0509/2/7

(C) 2008 Vazquez et al; licensee BioMed Central Ltd.

This is an Open Access article distributed under the terms of the Creative Commons Attribution License (http://creativecommons.org/licenses/by/2.0), which permits unrestricted use, distribution, and reproduction in any medium, provided the original work is properly cited.

\begin{abstract}
Background: Obtaining quantitative predictions for cellular metabolic activities requires the identification and modeling of the physicochemical constraints that are relevant at physiological growth conditions. Molecular crowding in a cell's cytoplasm is one such potential constraint, as it limits the solvent capacity available to metabolic enzymes.

Results: Using a recently introduced flux balance modeling framework (FBAwMC) here we demonstrate that this constraint determines a metabolic switch in $E$. coli cells when they are shifted from low to high growth rates. The switch is characterized by a change in effective optimization strategy, the excretion of acetate at high growth rates, and a global reorganization of $E$. coli metabolic fluxes, the latter being partially confirmed by flux measurements of central metabolic reactions.

Conclusion: These results implicate the solvent capacity as an important physiological constraint acting on E. coli cells operating at high metabolic rates and for the activation of a metabolic switch when they are shifted from low to high growth rates. The relevance of this constraint in the context of both the aerobic ethanol excretion seen in fast growing yeast cells (Crabtree effect) and the aerobic glycolysis observed in rapidly dividing cancer cells (Warburg effect) should be addressed in the future.
\end{abstract}

\section{Background}

Understanding an organism's metabolism at a system level requires knowledge of the physicochemical constraints limiting its metabolic capabilities under different growth conditions, and the genetic regulatory mecha- nisms that ultimately allow it to adapt to a changing environment. In some cases there is an obvious connection between an environmental change and the regulatory mechanisms responding to it, an example being a switch from aerobic to anaerobic growth [1]. However, there are 
constraints leading to less obvious metabolic changes, involving a complex global rearrangement of the cell's metabolism. A key aim of systems biology is to uncover the metabolic constraints determining such complex phenotypic changes, which can be understood only when the system is analyzed at a global scale [2-4].

In the absence of cell-scale kinetic models, flux balance analysis (FBA) provides experimentally testable predictions on an organism's metabolic flux state [4-8], which are based on conservation principles, particularly mass conservation, and metabolic capacity constraints. The impact of local constraints, such as uptake capacities, have been investigated [4-7], and capacity constraints over full metabolic pathways have been considered as well [9]. Moreover, it has been hypothesized that the high concentration of macromolecules in the cell's cytoplasm imposes a global constraint on the metabolic capacity of an organism $[10,11]$. More recently, we demonstrated that the key quantity is the total intracellular volume available to metabolic enzymes that result in a limited solvent capacity [12]. The addition of the solvent capacity constraint to a FBA model allowed us to explain, within a metabolic efficiency framework, the hierarchy of substrate consumption of $E$. coli cells growing in a mixture of carbon sources [12]. On the other hand, the pattern of substrate consumption can also be reproduced by superimposing regulatory information obtained e.g., from microarray data [13]. Taking together, these results indicate that the FBA model together with the solvent capacity constraint can be used to predict the regulatory mechanisms and, equally importantly, to understand their advantage in terms of metabolic efficiency and constraints. It is not clear, however, if the limited capacity constraint play a role at other physiological growth conditions, e.g., when nutrients are scarce.

Here we study the impact of the limited solvent capacity on E. coli cell metabolism at different physiological growth conditions. We demonstrate that this constraint is relevant for fast growing cells, and predict the existence of a metabolic switch between cells growing at low and high nutrient abundance, respectively. We carry out flux measurements of several reactions in the E. coli central metabolism, observing a partial agreement with the model predictions. Moreover, to uncover the regulatory mechanisms that control the changes in flux rates, we perform gene expression and enzyme activity measurements, finding that the switch is controlled predominantly at the enzyme activity level implemented by changes in the activity of a few key enzymes in the E. coli central metabolism. Finally, we discuss the potential relevance of the limited solvent capacity constraint to experimental observations in other organisms.

\section{Results \\ Limited solvent capacity constrains the metabolic rate of fast growing E. coli cells}

The cell's cytoplasm is characterized by a high concentration of macromolecules [14] resulting in a limited solvent capacity for the allocation of metabolic enzymes. More precisely, given that the enzyme molecules have a finite molar volume $v_{i}$ only a finite number of them fit in a given cell volume $V$. Indeed, if $n_{i}$ is the number of moles of the $i^{\text {th }}$ enzyme, then

$$
\sum_{i=1}^{N} v_{i} n_{i} \leq V
$$

where the inequality sign accounts for the volume of other cell components and the free volume necessary for cellular transport as well. Dividing by cell mass $M$ we can reformulate this inequality in terms of the enzyme concentrations $E_{i}=n_{i} / M$ (moles/unit mass), resulting in

$$
\sum_{i=1}^{N} v_{i} E_{i} \leq \frac{1}{C}
$$

where $C=M / V$ is the cytoplasmic density. An enzyme concentration $E_{i}$ results in a flux $f_{i}=b_{i} E_{i}$ over reaction $i$, where the parameter $b_{i}$ is determined by the reaction mechanism, kinetic parameters, and metabolite concentrations. Therefore, the enzyme concentration constraint (Eq. 2) is reflected in the metabolic flux constraint

$$
\sum_{i=1}^{N} a_{i} f_{i} \leq 1
$$

where

$$
a_{i}=\frac{C v_{i}}{b_{i}}
$$

Since the coefficients $a_{i}$ (units of inverse flux) quantifies the contribution to the overall crowding by reaction $i$ we refer to them as the 'crowding coefficients'.

To understand the relevance of the constraint (Eq. 3) at physiological growth conditions we first estimate the crowding coefficients (Eq. 4) using data from experimental reports. The E. coli cytoplasmic density of macromolecules is $C=0.34 \mathrm{~g} / \mathrm{ml}$ [15], while the molar volumes of proteins are proportional to their molar masses [16]. The coefficient of proportionality represents the specific volume and it is about $0.73 \mathrm{ml} / \mathrm{g}$. This empirical law allows us to compute the molar volumes of $E$. coli enzymes from their molar masses. As a first approximation we estimate $b_{i}$, the coefficient of proportionality between reaction rate and enzyme concentration, from the enzyme's turnover numbers. Data obtained from the BRENDA data base [17] 
for about hundred E. coli enzymes (Additional file 1) shows that the turnover numbers vary over five orders of magnitude (Fig. 1a), from $10^{-2}$ to $10^{2} 1 / \mathrm{s}$. Using these parameter estimates we compute the crowding coefficients $a_{i}$ for about a hundred E. coli enzymes (Fig. 1b), resulting in an average and standard deviation of 0.014 and $0.0091 /[\mathrm{mmol} / \mathrm{g} / \mathrm{h}]$, respectively. Because of the large enzyme turnover variations the crowding coefficients are distributed over a wide range as well, from $10^{-6}$ to $10^{0} 1 /[\mathrm{mmol} / \mathrm{g} / \mathrm{h}]$ (Fig. 1b).

\section{FBAwMC predicts a change of effective metabolic efficiency objective}

Having estimated the crowding coefficients we next evaluate the relevance of the solvent capacity constraint (Eq. 3 ) at physiological growth conditions. To this end we utilize a FBA model of E. coli MG1655 metabolic network that takes into account this constraint referred to as 'flux balance analysis with molecular crowding' (FBAwMC) [12]. Under conditions of aerobic growth in a glucoselimited medium, FBAwMC predicts a saturation of the glucose uptake rate and the growth rate (Fig. 2a,b) with increasing the glucose uptake capacity. The predicted maximum glucose uptake rate $(\sim 15 \mathrm{mmol} / \mathrm{g} / \mathrm{h})$ and maximum growth rate $\left(\sim 0.7 \mathrm{~h}^{-1}\right)$ are within the range of experimentally determined values [18], corroborating our previous report [12] that the solvent capacity constraint (Eq. 3) is relevant at physiological conditions.

Associated with the predicted saturation of E. coli metabolic rates, FBAwMC predicts a metabolic switch charac- terized by a change in the effective criteria of metabolic efficiency. At low growth rates the ratio between the biomass production rate and the glucose uptake rate is at a maximum but decreases with increasing the growth rate. In contrast, the ratio between the biomass production rate and the average reaction rate increases with increasing the growth rate, reaching a maximum at high growth rates. In agreement with our expectations, at low growth rates nutrients are scarce and the best strategy for a cell is to maximize the biomass production rate per unit of limiting nutrient (in this case, glucose) uptake rate. In contrast, at high growth rates the nutrients are abundant, the predicted metabolic rate is limited by the solvent capacity constraint (Eq. 3) and, therefore, the maximum growth rate is achieved by maximizing the biomass production rate per average reaction rate (Fig. 2c). The predicted change in metabolic efficiency objective is accompanied by a redistribution of the metabolic fluxes, including those of exchange fluxes. Indeed, a characteristic example is the predicted excretion of acetate at high growth rates (Fig. 2d) that is well supported by experimental observations $[9,19,20]$.

\section{FBAwMC-predicted metabolic fluxes are within the range of experimental values}

FBAwMC is also able to predict internal metabolic fluxes as a function of the growth rate. A subset of the FBAwMCderived flux predictions in the central carbon metabolism are shown in Figure 3. In most cases the FBAwMC predicted fluxes are within the range of experimentally determined values. This is a striking result given that this
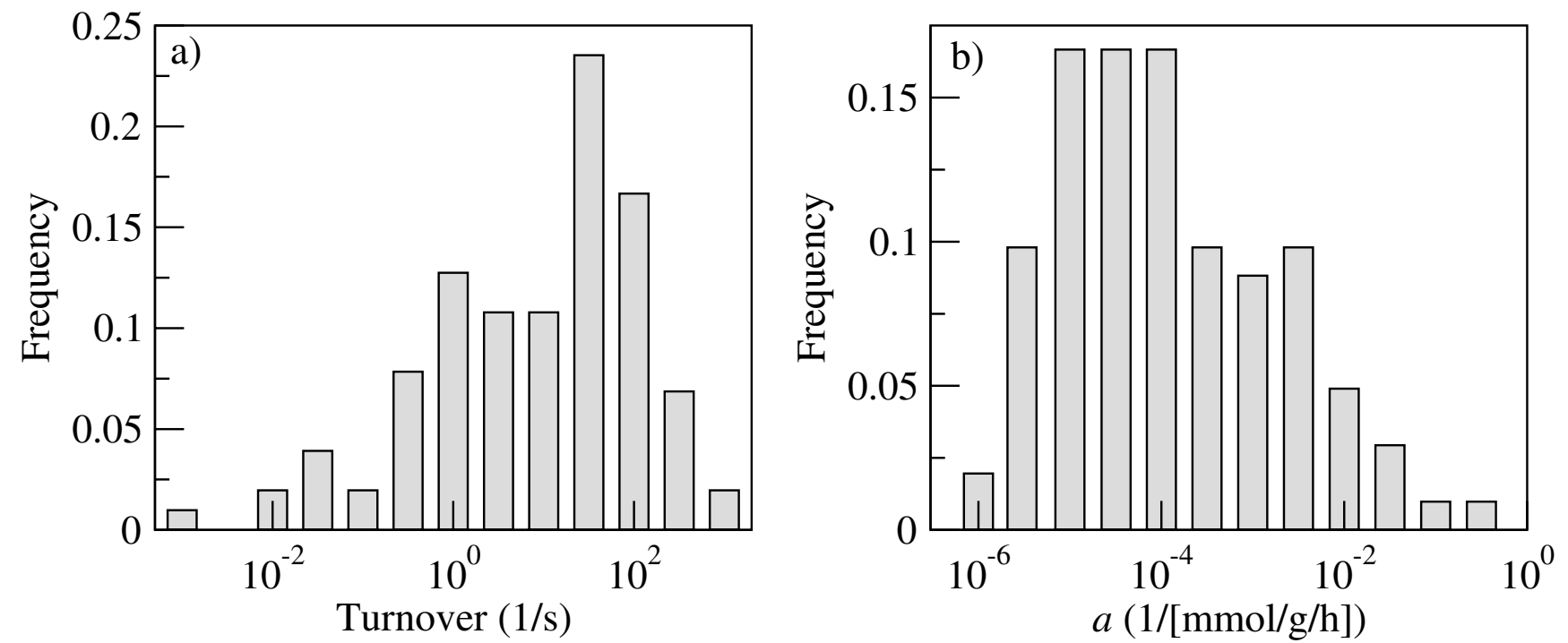

Figure I

Estimating the crowding coefficients of $E$. coli metabolic enzymes: (a) Distribution of turnover rates of $E$. coli enzymes as obtained from the BRENDA data base [17]; (b) Distribution of crowding coefficients among a hundred $E$. coli enzymes, as obtained using Eq. 4. 

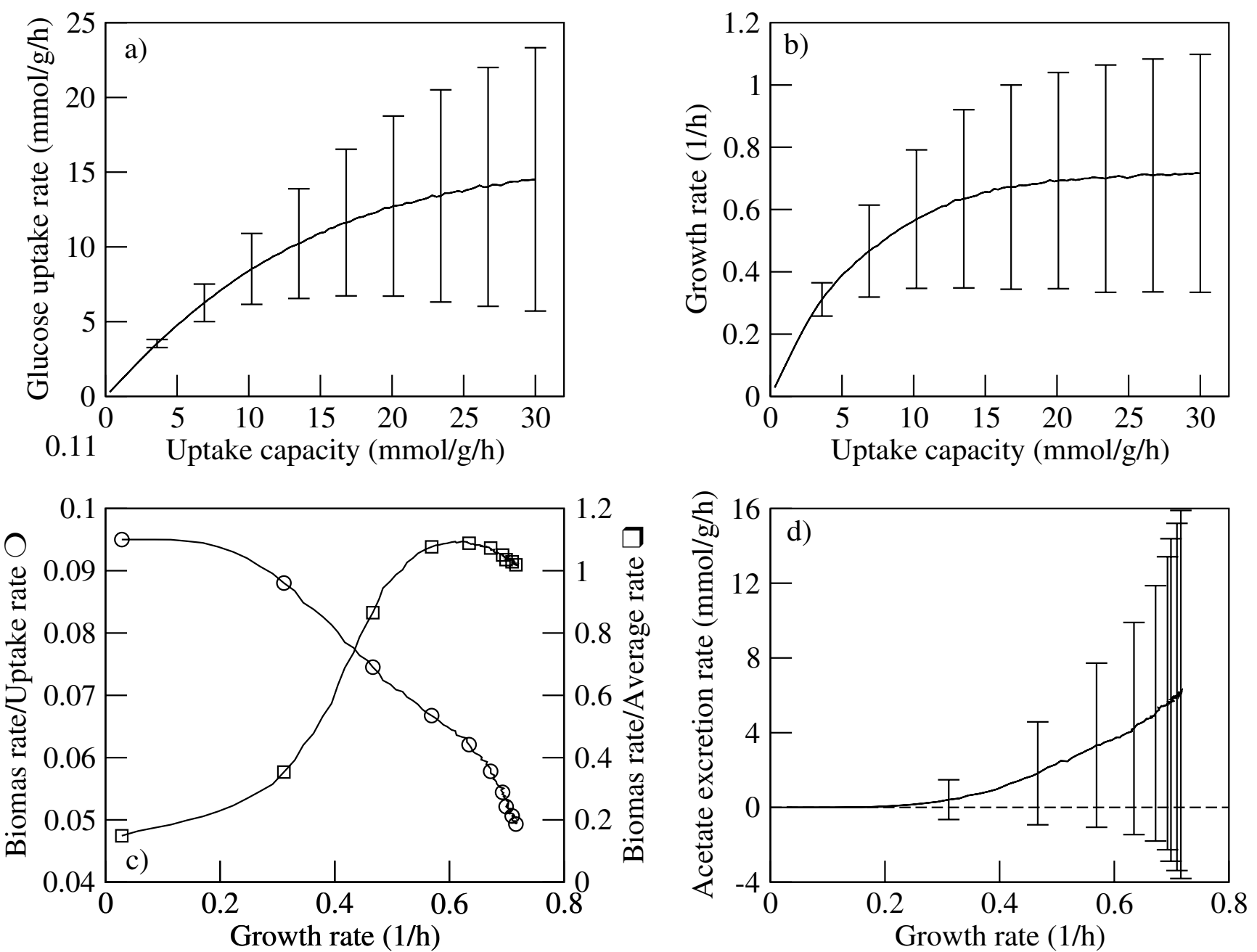

Figure 2

The signatures of the predicted metabolic switch: The glucose uptake rate (a) and growth rate (b) as a function of the glucose uptake capacity, as obtained from the FBAwMC model. The line represents the average behavior and the error bars represent the standard deviation over I,000 choices of crowding coefficients. (c) Flux ratios illustrating the switch in metabolic efficiency objective from low to high growth rates. At low growth the biomass rate per unit of uptake rate (circles) is at a maximum, while the biomass rate per unit of average rate is at a maximum at high growth rates (squares). (d) Acetate excretion rate as a function of the growth rate. At high growth rates the prediction for acetate excretion is sensitive to the crowding coefficients uncertainty, resulting in the large error bars.

implementation of FBAwMC does not contain any free paremeters. The only model parameters are the crowding coefficients, which were determined above using independent experimental results. We should also note that the observed wide variability around the average behavior (Fig. 3, experimental error bars) is not a shortcoming of our modeling framework but is due to our current inability to obtain a direct estimate for all crowding coefficients (due to incomplete information available on various kinetic parameters). Thus, further testing of our predictions will be necessary upon availability of better estimates for the crowding coefficients.
Limiting our analysis to the expected behavior, we observe a slope change for several fluxes when reaching the highest growth rates. The reactions of the glycolytic pathway, the flux towards the pentose-phosphate pathway via the reaction catalyzed by the gene product of $z w f$, and the acetate pathway switch at high growth rates to a faster flux increase with increasing the growth rate (Fig. 3). The experimental values corroborate this qualitative behavior, but the change is bigger for the $p t s G$-catalyzed reaction and even qualitatively different for the pykA-catalyzed reaction, both being part of the glycolytic pathway. A second noticeable effect is the predicted saturation of the 

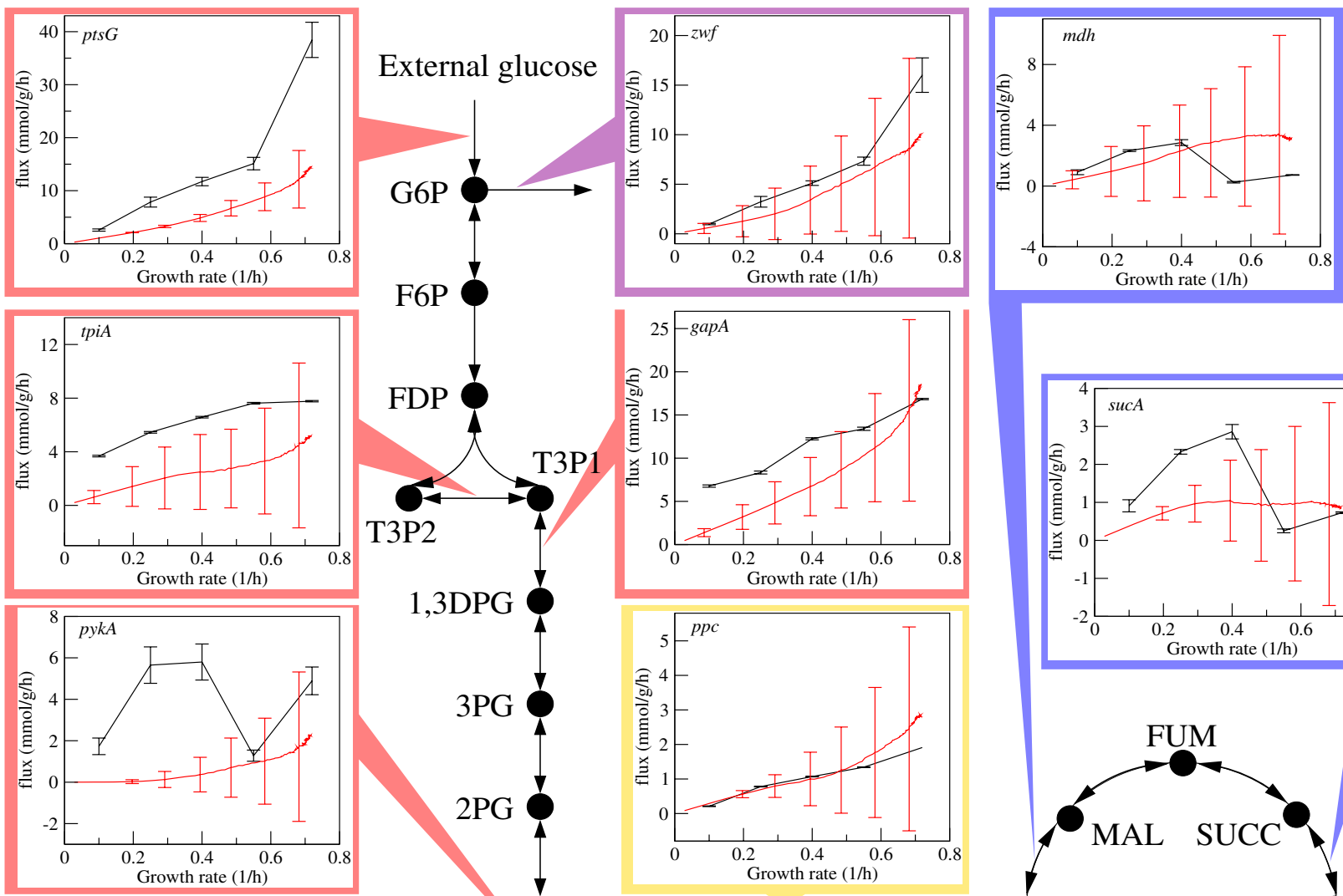

$1,3 \mathrm{DPG}$
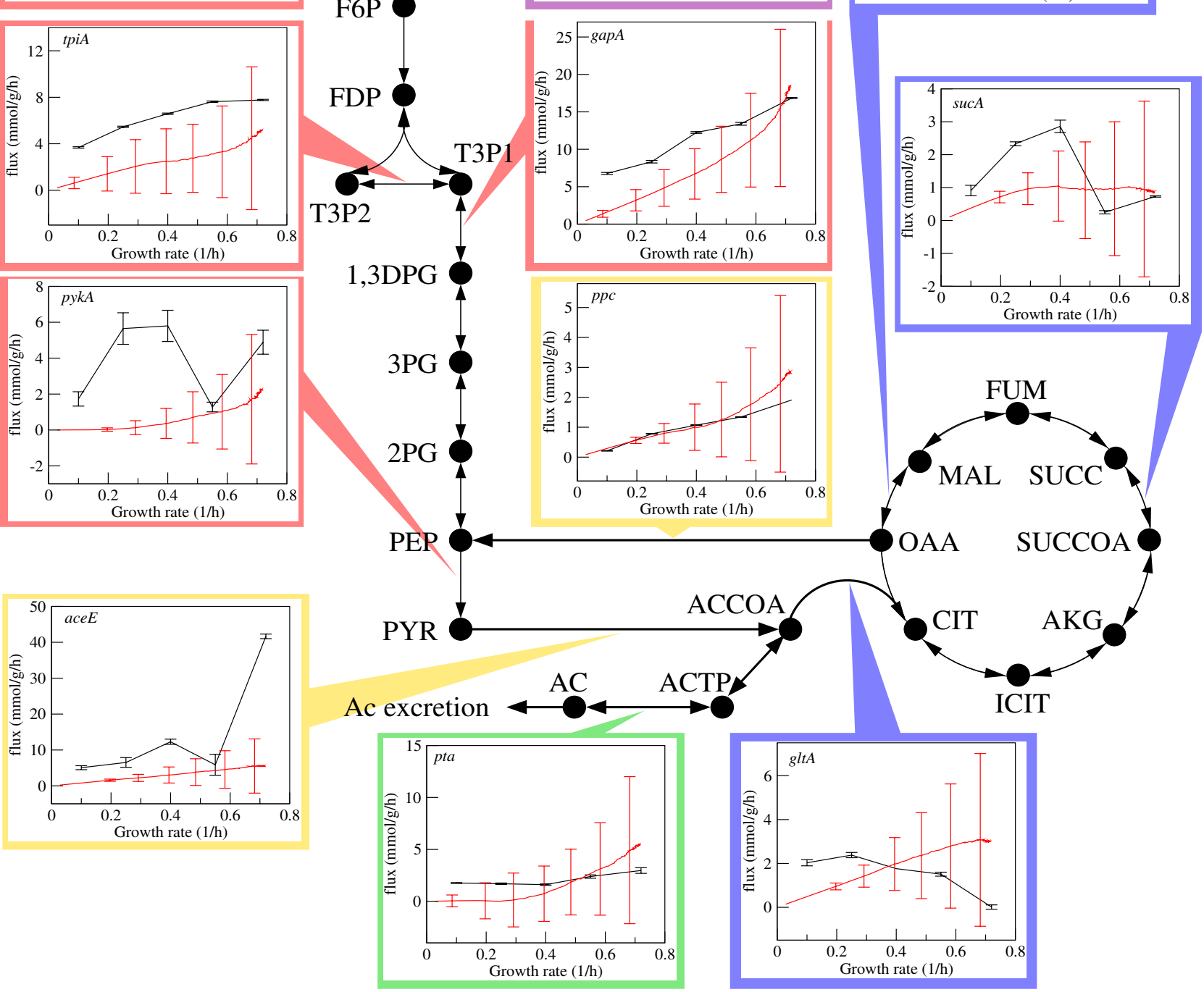

Figure 3

Predicted vs. measured metabolic fluxes in the E. coli central metabolism. Comparisons between the FBAwMC-predicted- (orange plots) and measured (blue plots) fluxes as a function of growth/dilution rates for selected reactions in the central carbon metabolism of $E$. coli. The experimental flux measurements were performed at dilution rates $0.1,0.25,0.4,0.55$ and $0.72 \mathrm{~h}^{-1}$. Selected reactions of glycolysis (red boxes), the first reaction of the pentose phosphate pathway (zwf) (magenta box), the TCA cycle (blue boxes), acetate excretion pathway (green boxes) and the reactions catalyzed by ppc and aceE connecting the glycolytic- and TCA pathways (yellow boxes) are shown. The solid black circles represent the denoted metabolites while the black arrows represent metabolic reactions labeled by the genes encoding the enzymes catalyzing the respective reactions (see Table SI in Additional file 4 for the list of abbreviations and information on enzymes encoded by listed genes). The error bars for predicted fluxes indicate the standard deviation over I,000 choices of the crowding coefficients among the list of values estimated for $\sim 100 \mathrm{E}$. coli enzymes, whereas the error bars for the experimental fluxes represent the standard deviations for three independent measurements. 
TCA cycle flux at high growth rates. The experimentally measured values of the TCA cycle flux exhibit, however, a stronger effect characterized by a decreasing tendency at high growth rates (Fig. 3). Taken together these results indicate that while for most reactions the FBAwMC predictions are within the range of experimental measurements, a method for a more accurate estimate of the crowding coefficients on a network scale will be required to provide more precise predictions.

\section{Identifying the regulatory mechanism(s) that control the action of the metabolic switch}

To examine if the changes in growth conditions and the corresponding adjustments in cellular metabolism can be traced by distinct molecular signatures we next measured the in vitro activity of eighteen selected enzymes (Additional file 2) that catalyze reactions in the central carbon metabolism of E. coli MG1655, and correlate their changes with those observed for the measured flux rates (Fig. 4). For several enzymes there is a good correlation between the measured enzyme- and flux activities (Pearson Correlation Coefficient, PCC, close to- or larger than $0.8)$. For example, with an increasing growth rate the enzyme activity of the $p t s G$ and $p f k A$ gene products follow the same increasing tendency as the fluxes of the corresponding metabolic reactions (PCC $=0.79$ and 0.85 , respectively). (The glycolytic flux is known to be controlled by the activity of these two enzymes while other reactions adjust their fluxes through changes in metabolite concentrations [21]). In contrast, we found no significant correlation between the measured fluxes and enzyme activities of the TCA reactions $($ PCC $=0.64,0.35,-0.03$ and -0.28 for enzymes associated with gltA, sucA, fumA and $m d h$, respectively), implying that the TCA flux is controlled by the activity of enzymes catalyzing reactions outside this pathway. A possible candidate to exert this action is the acetate pathway. Indeed, an increase of the flux on the acetate pathway towards the production of acetate can balance both the increase in the flux originating from the glycolytic pathway through aceE and a decrease in the flux from Acetyl-CoA to the TCA cycle. This hypothesis is supported by the increase in the enzyme activity of phosphotransacetylase ( $p t a)$ when the growth rate increases beyond $0.4 \mathrm{~h}^{-1}(\mathrm{PCC}=0.98)$, which is exactly the growth rate threshold where the switch is taking place.

In parallel with the enzyme activity measurements we also prepared mRNA from samples obtained at all five dilution rates and processed them for microarray analysis. The full microarray data set is presented in the Additional file 3 and its detailed analysis is presented in Additional file 4 (SI text 5-8). In contrast to the observed overall correlation between measured fluxes and in vitro enzyme activities we do not observe a significant correlation between the measured metabolic fluxes and the relative changes in
mRNA levels of enzyme-encoding genes (SI Fig. S5 in Additional file 4), implying that the switch and corresponding enzymatic functions are not predominantly controlled at the transcriptional level. Correspondingly, no significant correlation between the in vitro enzyme activities and the relative changes in mRNA levels of enzyme-encoding genes can be seen (SI Fig. S5 in Additional file 4). Taken together these results indicate that the metabolic switch is predominantly controlled by an increase in the enzyme activities of the end products of $p t s G$ and $p f k A$ controlling the glycolysis flux, and pta controlling the acetate pathway flux, respectively.

\section{Discussion}

Developing a modeling framework that can describe and predict in a quantitative manner the experimentally observed behavior of an organism is a significant challenge for systems biology. One prerequisite of this goal is to uncover the physicochemical constraints exerting the main influences on cellular metabolism [4]. Our results here and in Ref. [12] indicate that the limited solvent capacity represents a physiologically relevant constraint for fast growing E. coli cells. The incorporation of this constraint to the FBA modeling framework leads to the FBAwMC model whose predictions indicate that the solvent capacity constraint results in a maximum glucose uptake rate and growth rate that are within the range of experimentally determined values. The flux predictions for several reactions of the E. coli metabolism are within the range of our measurements, as well.

From the perspective of quantitative modeling using flux balance approximations, the solvent capacity constraint forces us to consider reaction kinetics via the crowding coefficients, at least for fast growing cells. At low metabolic rates the solvent capacity constraint is less relevant and flux balance alone is sufficient to obtain satisfactory predictions. In contrast, at high metabolic rates a precise knowledge of the crowding coefficients is required to obtain accurate predictions. In the absence of kinetic information we can still obtain a good approximation by sampling the crowding coefficients from a list of estimated values and then focus on the resulting general trend.

More importantly, the solvent capacity constraint allows the interpretation of the metabolic switch taking place between slow and fast growing $E$. coli cells. A recent study of FBA models with different objectives demonstrates that under nutrient scarcity a FBA model with the maximization of the biomass yield objective achieve the highest predictive accuracy, while maximizing the ATP or biomass yield per average flux unit is the best objective for unlimited growth on glucose under aerobic conditions [22]. In contrast, by considering the solvent capacity constraint we 


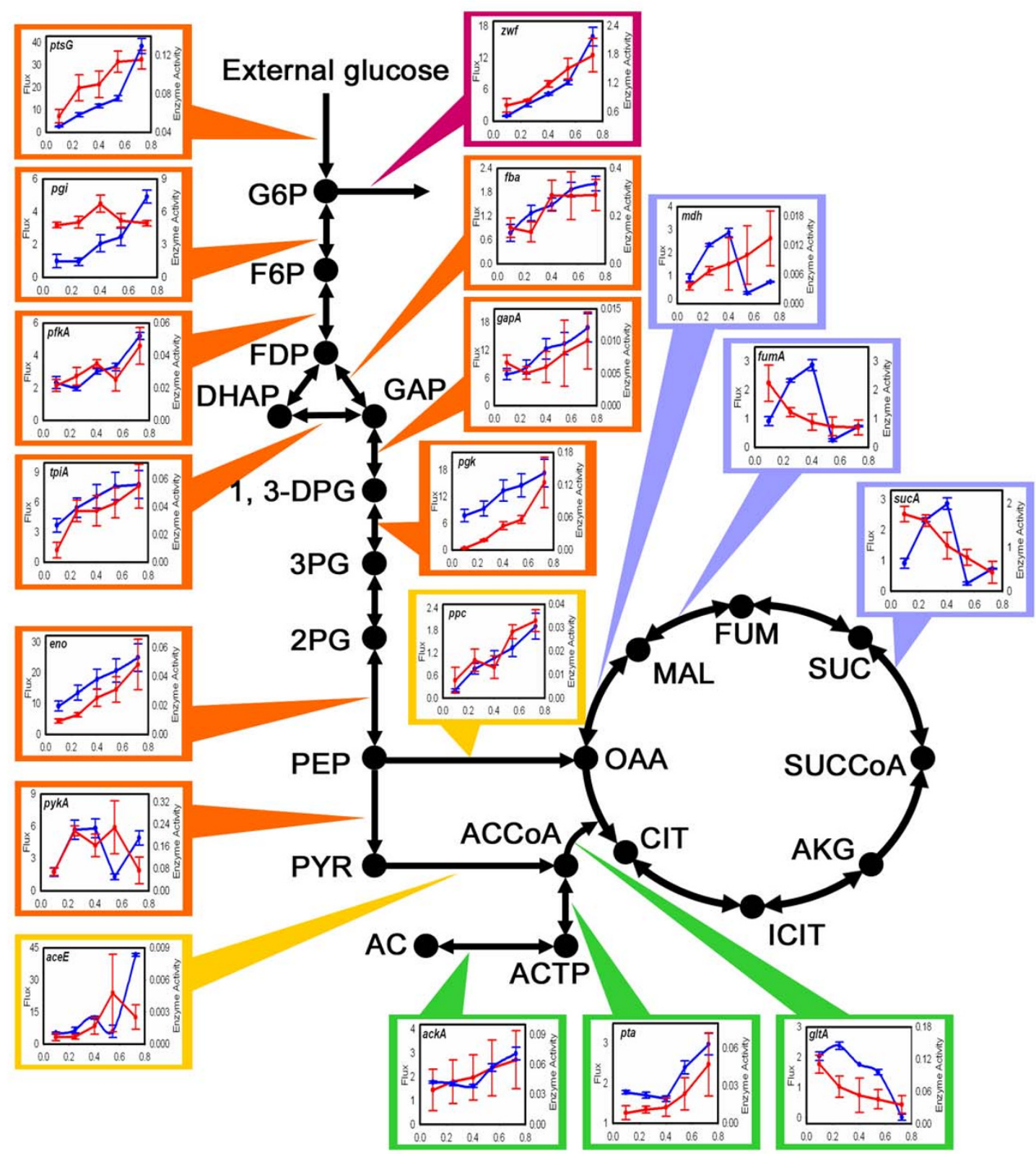

Figure 4

Comparison of measured metabolic fluxes and in-vitro enzyme activities. Measured flux rates (blue, $\mathrm{mmol} / \mathrm{h} / \mathrm{g} d r y$ biomass) and in vitro enzyme activities (red, $\mathrm{U} / \mathrm{mg}$ protein) on two separate $\mathrm{Y}$-axes of selected reactions in the central metabolism of $E$. coli are shown as a function of growth/dilution rates $(X$-axis). All labels are as in Figure 3. Additional file 2 shows the actual data on measured enzyme activities. The error bars for the experimental flux and enzyme activity plots are a result of three independent measurements. 
obtain the same results using the maximization of biomass production rate objective alone (Fig. $2 \mathrm{c}$ ). This is more consistent with the expectation that cells achieving the fastest growth rates outgrow cells growing at a slower rate, but how the highest growth rate is achieved is determined by both the availability of substrates and internal metabolic constraints, such as the solvent capacity. Furthermore, the well-known acetate excretion $[9,19,20]$ is explained by the solvent capacity constraint as well. We should note, however, that this does not exclude the possibility that under certain physiological conditions acetate excretion may result from a limited availability of oxygen in the culture medium [23].

\section{Conclusion}

From a more general perspective the results reported in this study further implicate the limited solvent capacity as an important constraint acting on the metabolism of cells operating at high metabolic rates. Thus, the relevance of this constraint will also need to be examined in the context of the observed aerobic ethanol excretion of fast growing yeast cells (Crabtree effect) [24] and aerobic glycolysis in fast growing mammalian cells, particularly tumor cells (Warburg effect) [25]. The common theme of these effects is that (i) they occur in fast growing cells and (ii) that in the presence of oxygen cells partially switch to anaerobic metabolism, resulting in the excretion of metabolic byproducts. Yet, the role of other proposed effects, such as a limit on attainable mitochondrial respiration [26] and available oxygen [23], cannot be excluded and, therefore, should be subject to further studies.

\section{Methods}

Estimation of crowding coefficients

The E. coli intracellular density is $C=0.34 \mathrm{~g} / \mathrm{ml}$ [15]. The specific volume was estimated for several proteins using the molar volumes and masses reported in Ref. [16], resulting in average of $0.73 \mathrm{ml} / \mathrm{g}$ and standard deviation of $0.02 \mathrm{ml} / \mathrm{g}$. The enzymes' turnover rates were obtained from the BRENDA database [17] for 102 E. coli enzymes.

\section{Metabolic flux predictions}

The Flux Balance analysis with Molecular Crowding [12] is implemented by solving the following optimization problem: maximize the biomass production rate subject to the constraints: balance in the production and consumption of each metabolite (flux balance), the maximum capacity constraint for the carbon source uptake rate and the solvent capacity constraint (Eq. 3). After expressing the reaction's stoichiometric coefficients in units of $\mathrm{mol} /$ dry biomass, the maximum growth rate corresponds to the biomass production rate, where biomass production is an auxiliary reaction containing as substrates the cellular components in their relative concentrations and as product the cell's biomass. The crowding coefficients were modeled as noise, assigning them randomly selected values from a list of estimated values for about hundred $E$. coli enzymes (Additional file 1). The predictions for all fluxes are provided in Additional file 5.

\section{Bacterial strain and general growth conditions}

The E. coli K12 strain MG1655 (F- $\lambda$ - ilvG rfb50 rph1) was used throughout the work. In order to obtain biomass samples for flux measurements, $20-\mathrm{ml}$ of the overnight grown culture ( $\sim 8-10 \mathrm{~h}$ ) of wild-type cells in LB-medium was inoculated in 980-ml M9 minimal medium (Sigma) containing $2 \mathrm{~g} / \mathrm{L}$ glucose, where $90 \%$ was natural glucose and the remaining $10 \%$ was labeled glucose $\left[1,2-{ }^{13} \mathrm{C}_{2}\right]-$ glucose (with $>99 \%$ purity and $99 \%$ isotope enrichment for each position, [Cambridge Isotope Laboratories, Andover, MA]). Cells were grown in a continuous growth mode at 5 different dilution rates $(0.1,0.25,0.4,0.55$, and $0.72 \mathrm{~L} \mathrm{~h}^{-1}$ ) in a Labfors bioreactor (Infors, Switzerland). The growth of the bacterial culture was regularly monitored at $\mathrm{A}_{600 \mathrm{~nm}}$ to document steady state, as described in Additional file 4 (SI text 3 and SI Fig. S7)

\section{Metabolic enzyme activity assays}

For determining metabolic enzyme activities from three separate experiments, the cell pellets (collected at all five dilution rates) were first resuspended and washed in 100 $\mathrm{mM}$ Tris-HCl ( $\mathrm{pH}$ 7.0) sonication buffer containing 20 $\mathrm{mM} \mathrm{KCl}, 5 \mathrm{mM} \mathrm{MnSO}{ }_{4}, 2 \mathrm{mM}$ DTT and $0.1 \mathrm{mM}$ EDTA, and then disrupted in a sonicator by 3 sonication cycles of 30 sec each. The cell debris was removed by centrifugation and the resulting cell extract (supernatant) was immediately used for enzyme assays or stored at $-20^{\circ} \mathrm{C}$. All subsequent steps were carried out on ice. Supernatants of the samples were used for quantitative assaying of endogenous enzyme activities by continuous spectrophotometric rate determination at $30^{\circ} \mathrm{C}$ in a thermostatically controlled spectrophotometer (Cary 500) with 1-cm light path, as described in the Additional file 4 (SI text 3). Supernatants of the samples were also used for determining total protein concentrations using standard Bradford's assay (BioRad).

\section{Flux measurements and analyses}

For flux analysis, biomass (from 100 ml culture) and supernatant samples were collected at all five dilution rates. These samples were immediately flash frozen in liquid nitrogen and stored at $-80^{\circ} \mathrm{C}$ until further analysis. Flux rates were determined using a tracer-substrate based GC-MS and NMR metabolome mapping platform. The analyses included determining positional ${ }^{13} \mathrm{C}$ tracer enrichment in multiple intermediary metabolites of glycolysis, glycogen synthesis, tricarboxylic acid cycle and their intracellular products from $\left[1,2-{ }^{13} \mathrm{C}_{2}\right]$-D-glucose, as described in detail in Additional file 4 (SI text 4 ). The retention times and mass-to-charge $(\mathrm{m} / \mathrm{z})$ ion clusters of 
selected ions of bacterial and culture media metabolites were determined using mass isotopomer analysis (MIDA) $[27,28]$, and expressed as net fluxes by subtracting reverse fluxes from forward tracer incorporation patterns via reversible metabolic steps [29]. Results were expressed as $\mathrm{mmol} / \mathrm{hr} / \mathrm{g}$ dry biomass glucose. Each experiment was carried out using triplicate cell cultures for each condition within each experiment, and the experiments were repeated once. Mass spectroscopic analyses were carried out by three independent automatic injections of $1 \mu \mathrm{l}$ samples by the automatic sampler and accepted only if the standard sample deviation was less than $1 \%$ of the normalized peak intensity. Statistical analysis was performed using the Student's t-test for unpaired samples. Two-tailed significance at the $99 \%$ confidence interval $(\mu+/-2.58 \sigma)$, $\mathrm{p}<0.01$ indicated significant differences in glucosederived fluxes. For some reversible reactions, we measured both forward and reverse fluxes and calculated net fluxes towards product synthesis.

\section{RNA preparation for microarray analysis}

At all five dilution rates, $10-20 \mathrm{ml}$ of the cell culture was collected, mixed with $10 \%(\mathrm{v} / \mathrm{v})$ of ice cold stop-solution ( $5 \%$ water-saturated phenol in absolute ethanol), and cell pellets were obtained by centrifugation at $4,500 \times g$ for 5 min at $4{ }^{\circ} \mathrm{C}$, followed by flash-freezing of pellets with liquid nitrogen. Cell pellets were stored at $-80^{\circ} \mathrm{C}$ until further use. RNA was isolated from the frozen cell pellets using Masterpure RNA isolation kit (Epicentre Biotechnologies, Madison, WI) and RNA samples were processed for transcriptome analysis using E. coli Affymetrix microarray chips, as described previously [12]. Dchip method was used to analyze all microarray data as described in Additional file 4 (SI text 5-8).

\section{Authors' contributions}

$\mathrm{AV}, \mathrm{MAM}$, and A-LB developed the theoretical framework and AV implemented it. QKB and LGB performed the experiments, with the exception of the microarray experiments that were performed at the Boston University Microarray Center. JE and ZB-J analyzed the microarray data. AV and ZNO guided and coordinated the project. $\mathrm{AV}, \mathrm{QKB}, \mathrm{ZB}-\mathrm{J}, \mathrm{LGB}$, and ZNO prepared the manuscript. All authors have read and approved the final version of the manuscript.

\section{Additional material}

\section{Additional file 1}

The list of reactions in E. coli in silico (iJE660a). From (http:// gcrg.ucsd.edu/organisms/ecoli/ecoli_reactions.html)

E. coli metabolic reactions and biomass vector

Click here for file

[http://www.biomedcentral.com/content/supplementary/17520509-2-7-S1.XLS]

\section{Additional file 2}

Measured enzyme activity values (U/mg protein) as shown in Fig. 4 (main manuscript) for various intracellular enzymes of the central carbon metabolism

Measured enzyme activity values for various intracellular enzymes of the central carbon metabolism

Click here for file

[http://www.biomedcentral.com/content/supplementary/17520509-2-7-S2.XLS]

\section{Additional file 3}

Dchip Microarray analysis of biomass samples from all five dilution/growth rates (Column H-L); actual hybridization intensities are in Columns B-F

Dchip Microarray analysis of biomass samples from all five dilution/ growth rates

Click here for file

[http://www.biomedcentral.com/content/supplementary/17520509-2-7-S3.XLS]

\section{Additional file 4}

Impact of the solvent capacity constraint on E. coli metabolism; Detailed supporting information, figs. 1-41

Impact of the solvent capacity constraint on $\mathrm{E}$. coli metabolism Click here for file

[http://www.biomedcentral.com/content/supplementary/17520509-2-7-S4.DOC]

\section{Additional file 5}

Flux predictions for 710 reactions in the in silico of E. coli metabolism during growth on glucose-limited medium.

Flux predictions for 710 reactions in the in silico of E. coli metabolism during growth on glucose-limited medium

Click here for file

[http://www.biomedcentral.com/content/supplementary/17520509-2-7-S5.XLS]

\section{Additional file 6}

List of all genes along with their ' $b$ '-numbers showing similar expression profile with query genes (green color) shown in SI Figs S14 to S36 in Supplementary Information

List of all genes along with their ' $b$ '-numbers showing similar expression profile with query genes.

Click here for file

[http://www.biomedcentral.com/content/supplementary/17520509-2-7-S6.XLS]

\section{Acknowledgements}

We thank N. Gerry and M. Lenburg at Boston University for their help with microarray experiments and A. Kochegarov at SiDMAP, LLC for metabolic flux measurements. Research at the University of Notre Dame and at the University of Pittsburgh was supported by National Institute of Health Grant \# U0I Al070499.

\section{References}

I. Kiley PJ, Beinert $\mathrm{H}$ : Oxygen sensing by the global regulator, FNR: the role of the iron-sulfur cluster. FEMS Microbiol Rev 1999, 22:34I-352. 
2. Hatzimanikatis V, Li C, lonita JA, Henry CS, Jankowski MD, Broadbelt LJ: Exploring the diversity of complex metabolic networks. Curr Opin Struct Biol 2004, 14:300-306.

3. Barabási AL, Oltvai ZN: Network biology: understanding the cell's functional organization. Nat Rev Genet 2004, 5: I0 I-I I 3 .

4. Price ND, Reed JL, Palsson BO: Genome-scale models of microbial cells: evaluating the consequences of constraints. Nat Rev Microbiol 2004, 2:886-897.

5. Beard DA, Liang SD, Qian H: Energy balance for analysis of complex metabolic networks. Biophys J 2002, 83:79-86.

6. Henry CS, Jankowski MD, Broadbelt LJ, Hatzimanikatis V: Genomescale thermodynamic analysis of Escherichia coli metabolism. Biophys J 2006, 90: 1453-1461.

7. Feist AM, Henry CS, Reed JL, Krummenacker M, Joyce AR, Karp PD, Broadbelt LJ, Hatzimanikatis V, Palsson BO: A genome-scale metabolic reconstruction for Escherichia coli K-I 2 MG I 655 that accounts for 1260 ORFs and thermodynamic information. Mol Syst Biol 2007, 3:121.

8. Segre $D$, Vitkup $D$, Church GM: Analysis of optimality in natural and perturbed metabolic networks. Proc Natl Acad Sci U S A 2002, 99(23): $15112-15117$

9. El-Mansi EM, Holms WH: Control of carbon flux to acetate excretion during growth of Escherichia coli in batch and continuous cultures. J Gen Microbiol 1989, 135:2875-2883.

10. Brown GC: Total cell protein-concentration as an evolutionary constraint on the metabolic control distribution in cells. J Theor Biol 1991, I 53:195-203.

II. Heinrich R, Schuster S: The regulation of cellular systems. New York, Chapman \& Hall; 1996.

12. Beg QK, Vázquez A, Ernst J, de Menezes MA, Bar-Joseph Z, Barabási $A L$, Oltvai $Z N$ : Intracellular crowding defines the mode and sequence of substrate uptake by Escherichia coli and constrains its metabolic activity. Proc Natl Acad Sci U S A 2007, 104: $12663-12668$

13. Covert MW, Famili I, Palsson BO: Identifying constraints that govern cell behavior: a key to converting conceptual to computational models in biology. Biotechnol Bioeng 2003, 84:763-772

14. Minton AP: How can biochemical reactions within cells differ from those in test tubes? J Cell Sci 2006, I I 9:2863-2869.

15. Zimmerman SB, Trach SO: Estimation of macromolecule concentrations and excluded volume effects for the cytoplasm of Escherichia coli. J Mol Biol I99|, 222:599-620.

16. Lee $B$ : Calculation of volume fluctuation for globular protein models. Proc Natl Acad Sci U S A 1983, 80:622-626.

17. Schomburg I, Chang A, Schomburg D: BRENDA, enzyme data and metabolic information. Nucleic Acids Res 2002, 30:47-49.

18. Fischer E, Zamboni N, Sauer U: High-throughput metabolic flux analysis based on gas chromatography-mass spectrometry derived I3C constraints. Anal Biochem 2004, 325:308-3I6.

19. Reiling HE, Laurila H, Fiechter A: Mass culture of Escherichia coli: medium development for low and high density cultivation of Escherichia coli B/r in minimal and complex media. J Biotechnol 1985, 2:191-206.

20. Wolfe AJ: The acetate switch. Microbiol Mol Biol Rev 2005, 69:12-50.

21. Cascante M, Boros LG, Boren J: Brain Energetics, Integration of Molecular and Cellular Processes. In Handbook of Neurochemistry and Molecular Neurobiology Edited by: Gibson GE, Dienel GA Springer; 2006.

22. Schuetz R, Kuepfer L, Sauer U: Systematic evaluation of objective functions for predicting intracellular fluxes in Escherichia coli. Mol Syst Biol 2007, 3:119.

23. Varma A, Palsson BO: Stoichimetric flux balance models quantitatively predict growth and metabolic by-product secretion in wild-type Escherichia coli W3 I I 0. Appl Environ Microbiol 1994, 60:3724-3731.

24. Frick O, Wittmann C: Characterization of the metabolic shift between oxidative and fermentative growth in Saccharomyces cerevisiae by comparative I3C flux analysis. Microb Cell Fact 2005, 4:30.

25. Chen Z, Lu W, Garcia-Prieto C, Huang P: The Warburg effect and its cancer therapeutic implications. J Bioenerg Biomembr 2007:ePub.

26. Vemuri GN, Eiteman MA, McEwen JE, Olsson L, Nielsen J: Increasing NADH oxidation reduces overflow metabolism in Sac- charomyces cerevisiae. Proc Natl Acad Sci U S A 2007, 104:2402-2407.

27. Lee WN, Byerley LO, Bassilian S, Ajie HO, Clark I, Edmond J, Bergner EA: Isotopomer study of lipogenesis in human hepatoma cells in culture: contribution of carbon and hydrogen atoms from glucose. Anal Biochem 1995, 226: I00-II2.

28. Lee WN, Edmond J, Bassilian S, Morrow JW: Mass isotopomer study of glutamine oxidation and synthesis in primary culture of astrocytes. Dev Neurosci 1996, 1 8:469-477.

29. Xu J, Lee WN, Xiao G, Trujillo C, Chang V, Blanco L, Hernandez F, Chung B, Makabi S, Ahmed S, Bassilian S, Saad M, Kurland IJ: Determination of a glucose-dependent futile recycling rate constant from an intraperitoneal glucose tolerance test. Anal Biochem 2003, 31 5:238-246.

Publish with BioMed Central and every scientist can read your work free of charge

"BioMed Central will be the most significant development for disseminating the results of biomedical research in our lifetime. "

Sir Paul Nurse, Cancer Research UK

Your research papers will be:

- available free of charge to the entire biomedical community

- peer reviewed and published immediately upon acceptance

- cited in PubMed and archived on PubMed Central

- yours - you keep the copyright 